\title{
Random ball-polyhedra and inequalities for intrinsic volumes
}

\author{
Grigoris Paouris* $\quad$ Peter Pivovarov ${ }^{\dagger}$
}

September 18, 2018

\begin{abstract}
We prove a randomized version of the generalized Urysohn inequality relating mean width to the other intrinsic volumes. To do this, we introduce a stochastic approximation procedure that sees each convex body $K$ as the limit of intersections of Euclidean balls of large radii and centered at randomly chosen points. The proof depends on a new isoperimetric inequality for the intrinsic volumes of such intersections. If the centers are i.i.d. and sampled according to a bounded continuous distribution, then the extremizing measure is uniform on a Euclidean ball. If one additionally assumes that the centers have i.i.d. coordinates, then the uniform measure on a cube is the extremizer. We also discuss connections to a randomized version of the extended isoperimetric inequality and symmetrization techniques.
\end{abstract}

\section{Introduction}

In this paper we discuss stochastic forms of classical inequalities for intrinsic volumes. Recall that the intrinsic volumes $V_{1}, \ldots, V_{n}$ are functionals on convex bodies which can be defined via the Steiner formula: for any convex body $K \subseteq$ $\mathbb{R}^{n}$ and $\varepsilon>0$,

$$
|K+\varepsilon B|=\sum_{j=0}^{n} \omega_{n-j} V_{j}(K) \varepsilon^{n-j},
$$

where $|\cdot|$ denotes $n$-dimensional Lebesgue measure, $B=B_{2}^{n}$ is the unit Euclidean ball in $\mathbb{R}^{n}, \omega_{n-j}$ is the volume of $B_{2}^{n-j}$, and $V_{0} \equiv 1 ; V_{1}$ is a multiple of the mean

\footnotetext{
${ }^{*}$ Grigoris Paouris is supported by the A. Sloan Foundation, US NSF grant CAREER-1151711 and BSF grant 2010288.

${ }^{\dagger}$ This work was partially supported by a grant from the Simons Foundation (\#317733 to Peter Pivovarov).

2010 Mathematics Subject Classification. Primary: 52A22, 52A40

Keywords and phrases. Convex body, mean width, Minkowski symmetrization, Steiner symmetrization, rearrangement inequalities, Wulff shape, generalized Urysohn inequality, intersections of congruent balls
} 
width $w, 2 V_{n-1}$ is the surface area and $V_{n}=|\cdot|$ is the volume. The $V_{j}$ 's satisfy the extended isoperimetric inequality: for $1 \leqslant j<n$,

$$
\left(\frac{V_{n}(K)}{V_{n}(B)}\right)^{1 / n} \leqslant\left(\frac{V_{j}(K)}{V_{j}(B)}\right)^{1 / j} ;
$$

as well as the generalized Urysohn inequality: for $1<j \leqslant n$,

$$
\left(\frac{V_{j}(K)}{V_{j}(B)}\right)^{1 / j} \leqslant \frac{V_{1}(K)}{V_{1}(B)} .
$$

The classical isoperimetric inequality corresponds to $j=n-1$ in (1.1); Urysohn's inequality to $j=n$ in (1.2) (or $j=1$ in (1.1)). The Alexandrov-Fenchel inequality for mixed volumes (e.g. [36]) implies both (1.1) and (1.2). Alternatively, symmetrization methods can be used. For example, Steiner symmetrization, which preserves $V_{n}(K)$ and does not increase $V_{j}(K)$, can be used to prove (1.1); a general framework for such inequalities, building on work of Rogers and Shephard [34], is discussed by Campi and Gronchi in [12]. On the other hand, Minkowski symmetrization, which fixes $V_{1}(K)$ and does not increase $V_{j}(K)$, can be used to prove (1.2); see [21, §6.4.4] ( $\$ 2$ contains definitions of these symmetrizations).

Both Steiner and Minkowski symmetrization can also be applied in suitable stochastic settings and yield stronger forms of such inequalities for associated random convex sets. For example, known inequalities for the expected intrinsic volumes of random convex hulls lead to (1.1). Such inequalities have their roots in the classical Sylvester's four point problem, e.g., [31], and build on work of Busemann [11], Groemer [19], Rogers-Shephard [34], Pfiefer [30], CampiGronchi [12], Hartzoulaki-Paouris [22], among others. Drawing on [28], one can formulate a type of stochastic dominance as follows. Assume that $|K|=|B|$ and sample independent random vectors $X_{1}, \ldots, X_{N}$ according to the uniform density $\frac{1}{|K|} \mathbb{1}_{K}$, i.e., $\mathbb{P}\left(X_{i} \in A\right)=\frac{1}{|K|} \int_{A} \mathbb{1}_{K}(x) d x$ for Borel sets $A \subseteq \mathbb{R}^{n}$. Additionally, sample independent random vectors $Z_{1}, \ldots, Z_{N}$ according to $\frac{1}{|B|} \mathbb{1}_{B}$. Then for all $1 \leqslant j \leqslant n$ and $s>0$,

$$
\mathbb{P}\left(V_{j}\left(\operatorname{conv}\left\{X_{1}, \ldots, X_{N}\right\}\right)>s\right) \geqslant \mathbb{P}\left(V_{j}\left(\operatorname{conv}\left\{Z_{1}, \ldots, Z_{N}\right\}\right)>s\right),
$$

where conv denotes the convex hull. Integrating in $s$ yields

$$
\mathbb{E} V_{j}\left(\operatorname{conv}\left\{X_{1}, \ldots, X_{N}\right\}\right) \geqslant \mathbb{E} V_{j}\left(\operatorname{conv}\left\{Z_{1}, \ldots, Z_{N}\right\}\right) .
$$

By the law of large numbers, the latter convex hulls converge to their respective ambient bodies and thus when $N \rightarrow \infty, V_{j}(K) \geqslant V_{j}(B)$ whenever $V_{n}(K)=V_{n}(B)$, 
which is equivalent to (1.1). Thus (1.1) can be seen as a global inequality which arises through a random approximation procedure in which stochastic domination holds at each stage. In fact, (1.3) holds not just for the convex hull but for a variety of other (linear, convex) operations and one can sample points according to continuous distributions on $\mathbb{R}^{n}$ (see [28]). Such distributional inequalities are useful for small deviation inequalities for the volume of random sets [29]; inequalities in the dual setting, obtained in joint work with Cordero-Erausquin and Fradelizi [14], lead to a stochastic version of the Blaschke-Santaló inequality and the $L_{p}$-versions of Lutwak and Zhang [26]. All of these inequalities concern volume and can be proved by Steiner symmetrization in an appropriate setting.

In a natural dual setting, Böröczky and Schneider [9] studied intersections of random halfspaces according to the following model. Let $\mathcal{H}$ denote the collection of all affine hyperplanes in $\mathbb{R}^{n}$ with its usual topology. Given a convex body $K$ with $V_{1}(K)=V_{1}(B)$, let $\mathcal{H}_{K}$ be the collection of $H \in \mathcal{H}$ that meet $K+B$ but do not intersect the interior of $K$. Let $\mu$ be the canonical motion invariant Borel measure on $\mathcal{H}$ normalized so that $\mu(\{H \in \mathcal{H}: H \cap M \neq \emptyset\})$ is the mean width $w(M)$ of $M$, for each convex body $M \subseteq \mathbb{R}^{n}$. Let $2 \mu_{K}$ be the restriction of $\mu$ to $\mathcal{H}_{K}$ so that $\mu_{K}$ is a probability measure. Sample independent hyperplanes $H_{1}, \ldots, H_{N}$ according to $\mu_{K}$ and $J_{1}, \ldots, J_{N}$ according to $\mu_{B}$. Denoting by $H_{i}^{-}$the closed halfspace bounded by $H_{i}$ and containing $K$, same for $J_{i}^{-}$and $B$, the following inequality holds for expectations

$$
\mathbb{E} V_{j}\left(\bigcap_{i=1}^{N} H_{i}^{-} \cap(K+B)\right)^{1 / j} \leqslant \mathbb{E} V_{j}\left(\bigcap_{i=1}^{N} J_{i}^{-} \cap(2 B)\right)^{1 / j}
$$

(their result is stated only for $j=1$ but the proof applies to all $1 \leqslant j \leqslant n$; the proof is reproduced in $\S 5$ ). When $N \rightarrow \infty$, one obtains $V_{j}(K) \leqslant V_{j}(B)$ whenever $V_{1}(K)=V_{1}(B)$, which is equivalent to (1.2). The proof of (1.5) uses Minkowski symmetrization. We are not aware of extensions of (1.5) to higher moments or for stochastic dominance.

In this paper we study a different model of random sets and a connection to (1.2) for which there is an underlying stochastic dominance. In [7], Bezdek, Lángi, Naszódi, and Papez study the intersection of finitely many (unit) Euclidean balls, called ball-polyhedra, and lay out a broad framework for their study; they treat analogues of classical theorems in convexity such as those of Carathéodory and Steinitz, and they study their facial structure. Motivation for their study arises, in part, from the Kneser-Poulsen Conjecture on the monotonicity of the volume of intersections (or unions) of Euclidean balls under contractions of their centers; see e.g. Bezdek's expository monograph [6]. We 
consider intersections of balls of a given radius $R>0$ with centers $X_{i}$ that are sampled independently according to a continuous distribution, i.e., a density $f: \mathbb{R}^{n} \rightarrow[0, \infty)$ with $\int_{\mathbb{R}^{n}} f(x) d x=1$ so that $\mathbb{P}\left(X_{i} \in A\right)=\int_{A} f(x) d x$ for Borel sets $A \subseteq \mathbb{R}^{n}$. In what follows, by a probability density we always mean that of a continuous distribution. Different random models associated with ball-polyhedra have been studied by Csikós [15], Ambrus, Kevei and Vígh [1] and Fodor, Kevei and Vígh [17].

Our first result is the following isoperimetric inequality for intrinsic volumes; here $B(x, r)$ is the closed Euclidean ball in $\mathbb{R}^{n}$ centered at $x \in \mathbb{R}^{n}$ with radius $r>0$ (so $B=B(0,1)$ ).

Theorem 1.1. Let $N, n \geqslant 1$ and $R>0$. Let $f$ be a probablity density on $\mathbb{R}^{n}$ that is bounded by one. Consider independent random vectors $X_{1}, \ldots, X_{N}$ sampled according to $f$ and $Z_{1}, \ldots, Z_{N}$ according to $\mathbb{1}_{B\left(0, r_{n}\right)}$ where $r_{n}>0$ is chosen so that $\left|B\left(0, r_{n}\right)\right|=1$. Then for all $1 \leqslant j \leqslant n$ and $s>0$,

$$
\mathbb{P}\left(V_{j}\left(\bigcap_{i=1}^{N} B\left(X_{i}, R\right)\right)>s\right) \leqslant \mathbb{P}\left(V_{j}\left(\bigcap_{i=1}^{N} B\left(Z_{i}, R\right)\right)>s\right) .
$$

For a particular choice of density $f,(1.6)$ can be seen as a form of (1.2) in which stochastic dominance holds. The connection arises from the following: given a convex body $K \subseteq \mathbb{R}^{n}$ with the origin in its interior and $K \subseteq B(0, R)$, define a star-shaped set $A(K, R)$ with radial function $\rho_{A(K, R)}(-\theta)=R-h_{K}(\theta)$ (see $\S 2$ for definitions). Euclidean balls centered at points in $A(K, R)$ are tangent to hyperplanes that meet $B(0, R)$ but not the interior of $K$. By choosing $f=$ $\frac{1}{|A(K, R)|} \mathbb{1}_{A(K, R)}$ in Theorem (1.1), we get the following corollary.

Corollary 1.2. Let $K$ be a convex body in $\mathbb{R}^{n}$ with the origin in its interior, $R>0$ and assume $K \subseteq B(0, R)$. Consider independent random vectors $X_{1}, \ldots, X_{N}$ sampled according to $\frac{1}{|A(K, R)|} \mathbb{1}_{A(K, R)}$ and $Z_{1}, \ldots, Z_{N}$ according to $\frac{1}{|r B|} \mathbb{1}_{r B}$, where $r=r(K, n, R)$ satisfies $|A(K, R)|=|r B|$. Then for each $p \in \mathbb{R}$,

$$
\left(\mathbb{E} V_{j}\left(\bigcap_{i=1}^{N} B\left(X_{i}, R\right)\right)^{p}\right)^{1 / p} \leqslant\left(\mathbb{E} V_{j}\left(\bigcap_{i=1}^{N} B\left(Z_{i}, R\right)\right)^{p}\right)^{1 / p} .
$$

For large $R$ the intersection of such balls resembles intersections of halfspaces; it turns out that the volume normalization $|A(K, R)|=|r B|$ amounts to a constraint on the mean width of $K$. When $N \rightarrow \infty$ and $R \rightarrow \infty$ in (1.7), we get (1.2). In fact, for fixed $N$, when $p \rightarrow-\infty$ and $R \rightarrow \infty$, we obtain the following result: among all convex bodies $K$ of a given mean width, the minimal $j$-th intrinsic volume of the intersection of $N>n$ halfspaces containing $K$ is maximized when $K$ is a ball; the latter is a special case of a result of Schneider [35], which is also proved using Minkowski symmetrization. 
The proof of Theorem 1.1 draws on both symmetrization techniques discussed above. We use the fact that $K \mapsto V_{j}(K)^{1 / j}$ is concave with respect to Minkowski addition, which follows by Minkowski symmetrization [21, §6.4.4], or the Alexandrov-Fenchel inequalities. However, using Steiner symmetrization and rearrangement inequalities, which are typically applied to volumetric inequalities, we obtain a distributional form of (1.2), which for $j<n$ is not a volumetric inequality. We make essential use of continuous distributions and intersections of Euclidean balls, as opposed to intersections of translates of other convex bodies or halfspaces (see Remark 3.7). Another fundamental ingredient in our proof is Kanter's theorem from [24] on stochastic dominance for products of unimodal densities, which we have not used before in this context. The techniques used in the proof of Theorem 1.1 also apply when $V_{j}$ is replaced by a function $\phi$ which is invariant under rotations, monotone and quasi-concave with respect to Minkowski addition (see Theorem 3.1).

As mentioned, (1.1) and (1.2) share a common result - Urysohn's inequality. We have discussed three randomized inequalities that have Urysohn's inequality as a consequence: for random convex hulls by taking $j=1$ in (1.4); for random halfspaces by taking $j=n$ in (1.5); for random ball-polyhedra by taking $j=n$ in (1.7). It is natural to investigate the relationship between the randomized forms. We note that the random ball-polyhedra version implies the random convex hull version. This is a consequence of a result of Gorbovickis [18], used to establish the Kneser-Poulsen conjecture for large radii (see §5).

We also consider random ball-polyhedra with independently chosen centers $X_{i}=\left(X_{i 1}, \ldots, X_{i n}\right) \in \mathbb{R}^{n}$ having independent coordinates and bounded densities, say by one. In this case, the uniform density on the unit cube $Q_{n}=[-1 / 2,1 / 2]^{n}$ is the extremizer.

Theorem 1.3. Let $N, n \geqslant 1$ and $R>0$. Let $h(x)=\prod_{i=1}^{n} h_{i}\left(x_{i}\right)$, where each $h_{i}$ is a probability density on $\mathbb{R}$ that is bounded by one. Consider independent random vectors $X_{1}, \ldots, X_{N}$ sampled according to $h$ and $Y_{1}, \ldots, Y_{N}$ according to $\mathbb{1}_{Q_{n}}$. Then for all $1 \leqslant j \leqslant n$ and $s>0$,

$$
\mathbb{P}\left(V_{j}\left(\bigcap_{i=1}^{N} B\left(X_{i}, R\right)\right)>s\right) \leqslant \mathbb{P}\left(V_{j}\left(\bigcap_{i=1}^{N} B\left(Y_{i}, R\right)\right)>s\right) .
$$

Lastly, on the organization of the paper: we recall definitions in $\$ 2$. Theorems 1.1 and 1.3 are proved in $\S 3$. In $\S 4$, we recall the definition of the Wulff shape and discuss a connection to (non-random) ball-polyhedra. In $\$ 5$, we prove Corollary 1.2 and compare it to kindred results for intersections of halfspaces, including a numerical improvement on the minimal volume simplex containing a convex body due to Kanazawa [23]; we also discuss Minkowski 
symmetrization, and compare two random versions of Urysohn's inequality.

\section{Preliminaries}

We work in Euclidean space $\mathbb{R}^{n}$ with the canonical inner product $\langle\cdot, \cdot\rangle$, Euclidean norm |.|; we also use |.| (or $V_{n}$ ) for volume. As above, the unit Euclidean ball in $\mathbb{R}^{n}$ is $B=B_{2}^{n}$ and its volume is $\omega_{n}:=\left|B_{2}^{n}\right| ; S^{n-1}$ is the unit sphere, equipped with the Haar probability measure $\sigma$.

A convex body $K \subseteq \mathbb{R}^{n}$ is a compact, convex set with non-empty interior. The set of all convex bodies in $\mathbb{R}^{n}$ is denoted by $\mathcal{K}^{n}$. For $K, L \in \mathcal{K}^{n}$, the Minkowski sum $K+L$ is the set $\{x+y: x \in K, y \in L\}$; for $\alpha>0, \alpha K=\{\alpha x: x \in K\}$. We say that $K$ is symmetric if it is symmetric about the origin, i.e., $-x \in K$ whenever $x \in K$. For $K \in \mathcal{K}^{n}$, the support function of $K$ is given by

$$
h_{K}(x)=\sup \{\langle y, x\rangle: y \in K\} \quad\left(x \in \mathbb{R}^{n}\right) .
$$

The mean width of $K$ is

$$
w(K)=\int_{S^{n-1}} h_{K}(\theta)+h_{K}(-\theta) d \sigma(\theta)=2 \int_{S^{n-1}} h_{K}(\theta) d \sigma(\theta) .
$$

If $K \in \mathcal{K}^{n}$ and $u \in S^{n-1}$, the Minkowski symmetral of $K$ about $u^{\perp}$ is the convex body

$$
M_{u}(K)=\frac{K+R_{u}(K)}{2},
$$

where $R_{u}$ is the reflection about $u^{\perp}$. The Steiner symmetral of a convex body will be defined later, and more generally for functions.

For compact sets $C_{1}, C_{2}$ in $\mathbb{R}^{n}$, we let $\delta^{H}\left(C_{1}, C_{2}\right)$ denote Hausdorff distance:

$$
\delta^{H}\left(C_{1}, C_{2}\right)=\inf \left\{\varepsilon>0: C_{1} \subseteq C_{2}+\varepsilon B_{2}^{n}, C_{2} \subseteq C_{1}+\varepsilon B_{2}^{n}\right\} .
$$

A set $K \subseteq \mathbb{R}^{n}$ is star-shaped if it is compact, contains the origin in its interior and for every $x \in K$ and $\lambda \in[0,1]$ we have $\lambda x \in K$. We call $K$ a star-body if its radial function

$$
\rho_{K}(\theta)=\sup \{s>0: s \theta \in K\} \quad\left(\theta \in S^{n-1}\right)
$$

is positive and continuous. Any positive continuous function $f: S^{n-1} \rightarrow \mathbb{R}$ determines a star body with radial function $f$.

For non-negative functions $f$ and $g$ on $[0, \infty)$, we write $f(r)=O(g(r))$ as $r \rightarrow \infty$ if there exists $M>0$ and $r_{0}>0$ such that $f(r) \leqslant M g(r)$ for all $r \geqslant r_{0}$; we write $f(r)=o(g(r))$ if $f(r) / g(r) \rightarrow 0$ as $r \rightarrow \infty$. 
We say that a non-negative function $f$ on $\mathbb{R}^{n}$ is quasi-concave if $\left\{x \in \mathbb{R}^{n}\right.$ : $f(x)>s\}$ is convex for each $s \geqslant 0$.

For Borel sets $A \subseteq \mathbb{R}^{n}$ with $|A|<\infty$, the volume-radius $\operatorname{vr}(A)$ is the radius of a Euclidean ball with the same volume as $A$; the symmetric rearrangement $A^{*}$ of $A$ is the (open) Euclidean ball of radius $\operatorname{vr}(A)$ centered at the origin. The symmetric decreasing rearrangement of $1_{A}$ is defined by $\left(1_{A}\right)^{*}:=1_{A^{*}}$. If $f: \mathbb{R}^{n} \rightarrow \mathbb{R}^{+}$ is an integrable function, we define its symmetric decreasing rearrangement $f^{*}$ by

$$
f^{*}(x)=\int_{0}^{\infty} 1_{\{f>s\}}^{*}(x) d s=\int_{0}^{\infty} 1_{\{f>s\}^{*}}(x) d s .
$$

The latter should be compared with the "layer-cake representation" of $f$ :

$$
f(x)=\int_{0}^{\infty} 1_{\{f>s\}}(x) d s ;
$$

see [25, Theorem 1.13]. The function $f^{*}$ is radially symmetric, radially decreasing (henceforth we simply say 'decreasing') and equimeasurable with $f$, i.e., $\{f>s\}$ and $\left\{f^{*}>s\right\}$ have the same volume for each $s>0$. By equimeasurability one has $\|f\|_{p}=\left\|f^{*}\right\|_{p}$ for each $1 \leqslant p \leqslant \infty$, where $\|\cdot\|_{p}$ denotes the $L_{p}\left(\mathbb{R}^{n}\right)$ norm. For a nonnegative, integrable function $f$ on $\mathbb{R}^{n}$, the rearrangement $f^{*}$ can be reached by a sequence of Steiner symmetrals $f^{*}(\cdot \mid \theta)$, which correspond to symmetrization in dimension one in the direction $\theta \in S^{n-1}$; namely $f^{*}(\cdot \mid \theta)$ is obtained by rearranging $f$ along every line parallel to $\theta$. The function $f^{*}(\cdot \mid \theta)$ is symmetric with respect to $\theta^{\perp}$. We refer the reader to the book [25] for further background material on rearrangements of functions.

\section{Extremal inequalities for random ball-polyhedra}

In this section we prove a more general version of Theorem 1.1. It concerns a family of functions $\phi: \mathcal{K}^{n} \rightarrow[0, \infty)$ satisfying the following three conditions:

(a) quasi-concave with respect to Minkowski addition: for all $K, L \in \mathcal{K}^{n}$ and $\lambda \in$ $(0,1)$

$$
\phi((1-\lambda) K+\lambda L) \geqslant \min (\phi(K), \phi(L)) ;
$$

(b) monotone: $\phi(K) \leqslant \phi(L)$ whenever $K, L \in \mathcal{K}^{n}$ satisfy $K \subseteq L$;

(c) rotation-invariant: $\phi(U K)=\phi(K)$ for all orthogonal transformations $U$ of $\mathbb{R}^{n}$ and $K \in \mathcal{K}^{n}$. 
The concavity of $K \mapsto V_{j}(K)^{1 / j}$ can be proved using Minkowski symmetrization as in $[21, \S 6.4 .4]$ or as a consequence of the Alexandrov-Fenchel inequalities; $V_{j}$ also satisfies $(b)$ and $(c)$ (see [36] for background).

Theorem 3.1. Let $N, n \geqslant 1$ and $r_{1}, \ldots, r_{N} \in(0, \infty)$. Assume that $\phi: \mathcal{K}^{n} \rightarrow[0, \infty)$ satisfies $(a)$, (b) and (c). Let $f_{1}, \ldots, f_{N}$ be probability densities on $\mathbb{R}^{n}$. Consider independent random vectors $X_{1}, \ldots, X_{N}$ and $X_{1}^{*}, \ldots, X_{N}^{*}$ such that $X_{i}$ is distributed according to $f_{i}$ and $X_{i}^{*}$ according to $f_{i}^{*}$, for $i=1, \ldots, N$. Then for any $s \geqslant 0$,

$$
\mathbb{P}\left(\phi\left(\bigcap_{i=1}^{N} B\left(X_{i}, r_{i}\right)\right)>s\right) \leqslant \mathbb{P}\left(\phi\left(\bigcap_{i=1}^{N} B\left(X_{i}^{*}, r_{i}\right)\right)>s\right) .
$$

Furthermore, assume each $f_{i}$ is bounded. Let $Z_{1}, \ldots, Z_{N}$ be independent random vectors with $Z_{i}$ distributed according to $a_{i} \mathbb{1}_{b_{i} B}$, where $a_{i}=\left\|f_{i}\right\|_{\infty}$ and $b_{i}$ satisfies $\int_{\mathbb{R}^{n}} a_{i} \mathbb{1}_{b_{i} B} d x=1$, for $i=1, \ldots, N$. Then

$$
\mathbb{P}\left(\phi\left(\bigcap_{i=1}^{N} B\left(X_{i}, r_{i}\right)\right)>s\right) \leqslant \mathbb{P}\left(\phi\left(\bigcap_{i=1}^{N} B\left(Z_{i}, r_{i}\right)\right)>s\right) .
$$

As in [28], [29], we use the rearrangement inequality of Rogers [33] and Brascamp-Lieb-Luttinger [10]; in particular, the following variant due to Christ [13].

Theorem 3.2. Let $F:\left(\mathbb{R}^{n}\right)^{N}=\otimes_{i=1}^{N} \mathbb{R}^{n} \rightarrow[0, \infty)$. Then

$$
\begin{aligned}
\int_{\left(\mathbb{R}^{n}\right)^{N}} F\left(x_{1}, \ldots, x_{N}\right) f_{1}\left(x_{1}\right) & \cdots f_{N}\left(x_{N}\right) d x_{1} \ldots d x_{N} \\
& \leqslant \int_{\left(\mathbb{R}^{n}\right)^{N}} F\left(x_{1}, \ldots, x_{N}\right) f_{1}^{*}\left(x_{1}\right) \cdots f_{N}^{*}\left(x_{N}\right) d x_{1} \ldots d x_{N}
\end{aligned}
$$

holds for any integrable $f_{1}, \ldots, f_{N}: \mathbb{R}^{n} \rightarrow[0, \infty)$ whenever $F$ satisfies the following condition: for every $z \in S^{n-1} \subseteq \mathbb{R}^{n}$ and for every $Y=\left(y_{1}, \ldots, y_{N}\right) \subseteq\left(z^{\perp}\right)^{N} \subseteq\left(\mathbb{R}^{n}\right)^{N}$, the function $F_{z, Y}: \mathbb{R}^{N} \rightarrow[0, \infty)$ defined by

$$
F_{z, Y}(t):=F\left(y_{1}+t_{1} z, \ldots, y_{N}+t_{N} z\right) .
$$

is even and quasi-concave.

Remark 3.3. (i) When $n=1$, the condition on $F$ in the latter theorem reduces to $F: \mathbb{R}^{N} \rightarrow[0, \infty)$ being even and quasi-concave.

(ii) The proof of the latter theorem relies on the fact that such integrals are increased when the $f_{i}$ 's are replaced by their Steiner symmetrals $f_{i}^{*}(\cdot \mid \theta)$. 
When repeated in suitable directions $\theta$, they yield the symmetric decreasing rearrangements $f_{i}^{*}$. We refer the reader to [13] or [28], [14] for the details.

We also combine the latter with a theorem of Kanter [24, Corollary 3.2]. If $f$ and $g$ are probability densities on $\mathbb{R}^{n}$ such that $\int_{K} f(x) d x \leqslant \int_{K} g(x) d x$ for every symmetric convex set $K \subseteq \mathbb{R}^{n}$, we will use similar terminology as that used in [4], [5], [3] and say that $f$ is less peaked than $g$ (here, as above, 'symmetric' means 'origin-symmetric'). Furthermore, we say that $f$ is unimodal if it is quasi-concave and even. Kanter uses a more general notion of unimodality but his result applies to the condition we use here; see the discussion in [24, §5].

Theorem 3.4. Let $n, N \geqslant 1$. Let $f_{1}, \ldots, f_{N}$ and $g_{1}, \ldots, g_{N}$ be unimodal probability densities on $\mathbb{R}^{n}$. Assume that $f_{i}$ is less peaked than $g_{i}$ for each $i=1, \ldots, N$. Then $\prod_{i=1}^{n} f_{i}$ is less peaked than $\prod_{i=1}^{n} g_{i}$.

We will also use the following basic lemma (it can be proved using, e.g., [14, Lemma 4.3]).

Lemma 3.5. Any radial probability density on $\mathbb{R}^{n}, n \geqslant 1$, that is bounded by one is less peaked than $\mathbb{1}_{B\left(0, r_{n}\right)}$ where $r_{n}$ satisfies $\left|B\left(0, r_{n}\right)\right|=1$; in particular, taking $n=$ 1 , any even probability density on $\mathbb{R}$ that is bounded by one is less peaked than $\mathbb{1}_{[-1 / 2,1 / 2]}$.

The requisite quasi-concavity needed to apply Theorem 3.2 is a consequence of the following lemma.

Lemma 3.6. Let $N, n \geqslant 1$ and $r_{1}, \ldots, r_{N} \in(0, \infty)$. Assume that $\phi: \mathcal{K}^{n} \rightarrow[0, \infty)$ satisfies $(a)$ and $(b)$ and $\phi(K)=\phi(-K)$ for each $K \in \mathcal{K}^{n}$. Set

$$
F\left(x_{1}, \ldots, x_{N}\right)=\phi\left(\bigcap_{i=1}^{N} B\left(x_{i}, r_{i}\right)\right) .
$$

Then $F$ is even and quasi-concave on its support. Additionally, assume that $\phi$ satisfies condition (c). If $z \in S^{n-1}$ and $y_{1}, \ldots, y_{N} \in z^{\perp}$ and $F_{z, Y}: \mathbb{R}^{N} \rightarrow[0, \infty)$ is defined by

$$
F_{z, Y}(t):=\phi\left(\bigcap_{i=1}^{N} B\left(y_{i}+t_{i} z, r_{i}\right)\right),
$$

then $F_{z, Y}$ is even and quasi-concave on its support.

Proof. The function $F$ is clearly even on $\left(\mathbb{R}^{n}\right)^{N}$. For the quasi-concavity claim, let $\mathbf{u}=\left(u_{1}, \ldots, u_{N}\right) \in\left(\mathbb{R}^{n}\right)^{N}$ and $\mathbf{v}=\left(v_{1}, \ldots, v_{N}\right) \in\left(\mathbb{R}^{n}\right)^{N}$ belong to the support of $F$. We will first show that

$$
\bigcap_{i=1}^{N} B\left(\frac{u_{i}+v_{i}}{2}, r_{i}\right) \supseteq \frac{1}{2} \bigcap_{i=1}^{N} B\left(u_{i}, r_{i}\right)+\frac{1}{2} \bigcap_{i=1}^{N} B\left(v_{i}, r_{i}\right) .
$$


Let $w_{1}, w_{2} \in \mathbb{R}^{n}$ and assume $\left|w_{1}-u_{i}\right| \leqslant r_{i}$ and $\left|w_{2}-v_{i}\right| \leqslant r_{i}$ for $i=1, \ldots, N$. Then for $i=1, \ldots, N$,

$$
\left|\frac{w_{1}+w_{2}}{2}-\left(\frac{u_{i}+v_{i}}{2}\right)\right| \leqslant \frac{1}{2}\left|w_{1}-u_{i}\right|+\frac{1}{2}\left|w_{2}-v_{i}\right| \leqslant r_{i}
$$

which shows the inclusion. By monotonicity and quasi-concavity of $\phi$, we have

$$
\begin{aligned}
F((\mathbf{u}+\mathbf{v}) / 2) & =\phi\left(\bigcap_{i=1}^{N} B\left(\frac{u_{i}+v_{i}}{2}, r_{i}\right)\right) \\
& \geqslant \phi\left(\frac{1}{2} \bigcap_{i=1}^{N} B\left(u_{i}, r_{i}\right)+\frac{1}{2} \bigcap_{i=1}^{N} B\left(v_{i}, r_{i}\right)\right) \\
& \geqslant \min \left(\phi\left(\bigcap_{i=1}^{N} B\left(u_{i}, r_{i}\right)\right), \phi\left(\bigcap_{i=1}^{N} B\left(v_{i}, r_{i}\right)\right)\right) \\
& =\min (F(\mathbf{u}), F(\mathbf{v})) .
\end{aligned}
$$

Therefore, $F$ is quasi-concave on its support.

The second quasi-concavity claim follows from the fact that the restriction of a quasi-concave function to a line is itself quasi-concave. Finally, let $z \in S^{n-1}$ and $y_{1}, \ldots, y_{N} \in z^{\perp}$. Let $R_{z}$ denote the reflection about $z^{\perp}$. Then

$$
\begin{aligned}
R_{z}\left(\bigcap_{i=1}^{N} B\left(y_{i}+t_{i} z, r_{i}\right)\right) & =\bigcap_{i=1}^{N} R_{z}\left(r_{i} B(0,1)+\left(y_{i}+t_{i} z\right)\right) \\
& =\bigcap_{i=1}^{N}\left(r_{i} B(0,1)+\left(y_{i}-t_{i} z\right)\right) \\
& =\bigcap_{i=1}^{N} B\left(y_{i}-t_{i} z, r_{i}\right) .
\end{aligned}
$$

Since $\phi$ satisfies $(c)$, we have

$$
F_{z, Y}(t)=\phi\left(\bigcap_{i=1}^{N} B\left(y_{i}+t_{i} z, r_{i}\right)\right)=\phi\left(\bigcap_{i=1}^{N} B\left(y_{i}-t_{i} z, r_{i}\right)\right)=F_{z, Y}(-t) .
$$

Remark 3.7. In the previous lemma, the use of Euclidean balls is not important to obtain quasi-concavity of $F$; one can also take intersections of translates of other convex bodies. However, to obtain the evenness condition on $F_{z, Y}$ it is essential that we use Euclidean balls. Thus ball-polyhedra interface well with the rearrangement techniques used here, as the next proof shows.

Proof of Theorem 3.1. Let $F$ be as in Lemma 3.6. For $s>0$, set $H=\mathbb{1}_{\{F>s\}}$. Let $z \in S^{n-1}$ and $Y=\left(y_{1}, \ldots, y_{N}\right) \in\left(z^{\perp}\right)^{N}$. Let $F_{z, Y}\left(t_{1}, \ldots, t_{N}\right)=F\left(y_{1}+t_{1} z, \ldots, y_{N}+t_{N} z\right)$ 
and $H_{z, Y}\left(t_{1}, \ldots, t_{N}\right)=\mathbb{1}_{\left\{F_{z, Y}>s\right\}}\left(t_{1}, \ldots, t_{N}\right)$. By Lemma 3.6, $F_{z, Y}$ is an even, quasiconcave function. It follows that $H_{z, Y}$ is even and quasi-concave. Therefore we can apply Theorem 3.2 to obtain

$$
\begin{aligned}
\mathbb{P}( & \left.\phi\left(\bigcap_{i=1}^{N} B\left(X_{i}, r_{i}\right)\right)>s\right) \\
& =\int_{\mathbb{R}^{n}} \ldots \int_{\mathbb{R}^{n}} H\left(x_{1}, \ldots, x_{N}\right) \prod_{i=1}^{N} f_{i}\left(x_{i}\right) d x_{1} \ldots d x_{N} \\
& \leqslant \int_{\mathbb{R}^{n}} \ldots \int_{\mathbb{R}^{n}} H\left(x_{1}, \ldots, x_{N}\right) \prod_{i=1}^{N} f_{i}^{*}\left(x_{i}\right) d x_{1} \ldots d x_{N} \\
& =\mathbb{P}\left(\phi\left(\bigcap_{i=1}^{N} B\left(X_{i}^{*}, r_{i}\right)\right)>s\right),
\end{aligned}
$$

which proves (3.1).

We will first prove (3.2) under the additional assumption that $\left\|f_{i}\right\|_{\infty}=1$ for $i=1, \ldots, N$. Furthermore, by the first part of the proof we may assume that each $f_{i}$ is radially symmetric and radially decreasing, hence unimodal. By Lemma 3.5, $f_{i}$ is less peaked than $\mathbb{1}_{B\left(0, r_{n}\right)}$. Since $H=\mathbb{1}_{\{F>s\}}$ is the indicator function of a symmetric convex set in $\left(\mathbb{R}^{n}\right)^{N}$, Theorem 3.4 yields

$$
\begin{aligned}
\mathbb{P}( & \left.\phi\left(\cap_{i=1}^{N} B\left(X_{i}, r_{i}\right)\right)>s\right) \\
\quad & =\int_{\mathbb{R}^{n}} \ldots \int_{\mathbb{R}^{n}} H\left(x_{1}, \ldots, x_{N}\right) \prod_{i=1}^{N} f_{i}\left(x_{i}\right) d x_{1} \ldots d x_{N} \\
\quad & \leqslant \int_{\mathbb{R}^{n}} \ldots \int_{\mathbb{R}^{n}}^{N} H\left(x_{1}, \ldots, x_{N}\right) \prod_{i=1}^{N} \mathbb{1}_{B\left(0, r_{n}\right)}\left(x_{i}\right) d x_{1} \ldots d x_{N} \\
& =\mathbb{P}\left(\phi\left(\bigcap_{i=1}^{N} B\left(Z_{i}, r_{i}\right)\right)>s\right) .
\end{aligned}
$$

The general case follows by a change of variables; note that we make no assumption of homogeneity of $\phi$ in the following argument. For $i=1, \ldots, N$, let $c_{i}=\left\|f_{i}\right\|_{\infty}^{-1 / n}$ and set

$$
\bar{f}_{i}(x)=\frac{f_{i}\left(c_{i} x\right)}{\int_{\mathbb{R}^{n}} f_{i}\left(c_{i} y\right) d y}=\frac{f_{i}\left(c_{i} x\right)}{\left\|f_{i}\right\|_{\infty}} .
$$

Then $\left\|\bar{f}_{i}\right\|_{1}=\left\|\bar{f}_{i}\right\|_{\infty}=1$ for $i=1, \ldots, N$. We apply what we just proved with $\bar{f}_{1}, \bar{f}_{2}, \ldots, \bar{f}_{N}$ and $H\left(c_{1}, \ldots, c_{N} \cdot\right)$ (which remains the indicator of a symmetric convex set)

$$
\mathbb{P}\left(\phi\left(\bigcap_{i=1}^{N} B\left(X_{i}, r_{i}\right)\right)>s\right)
$$




$$
\begin{aligned}
& =\int_{\mathbb{R}^{n}} \ldots \int_{\mathbb{R}^{n}} H\left(x_{1}, \ldots, x_{N}\right) \prod_{i=1}^{n} f_{i}\left(x_{i}\right) d x_{1} \ldots d x_{N} \\
& =\prod_{i=1}^{N}\left\|f_{i}\right\|_{\infty} \int_{\mathbb{R}^{n}} \ldots \int_{\mathbb{R}^{n}} H\left(c_{1} y_{1}, \ldots, c_{n} y_{N}\right) \prod_{i=1}^{N} \frac{c_{i}^{n} f_{i}\left(c_{i} y_{i}\right)}{\left\|f_{i}\right\|_{\infty}} d y_{1} \ldots d y_{N} \\
& =\int_{\mathbb{R}^{n}} \ldots \int_{\mathbb{R}^{n}} H\left(c_{1} y_{1}, \ldots, c_{n} y_{N}\right) \prod_{i=1}^{N} \bar{f}_{i}\left(y_{i}\right) d y_{1} \ldots d y_{N} \\
& \leqslant \int_{\mathbb{R}^{n}} \ldots \int_{\mathbb{R}^{n}} H\left(c_{1} y_{1}, \ldots, c_{n} y_{N}\right) \prod_{i=1}^{N} \mathbb{1}_{r_{n} B}\left(y_{i}\right) d y_{1} \ldots d y_{N} \\
& =\int_{\mathbb{R}^{n}} \ldots \int_{\mathbb{R}^{n}}^{N} H\left(x_{1}, \ldots, x_{N}\right) \prod_{i=1}^{N}\left\|f_{i}\right\|_{\infty} \mathbb{1}_{c_{i} r_{n} B}\left(x_{i}\right) d x_{1} \ldots d x_{N} \\
& =\mathbb{P}\left(\phi\left(\bigcap_{i=1}^{N} B\left(Z_{i}, r_{i}\right)\right)>s\right),
\end{aligned}
$$

where, as above, $r_{n}=\omega_{n}^{-1 / n}$. This proves (3.2) as claimed with $b_{i}=c_{i} r_{n}$, for $i=1, \ldots, N$.

Now we turn to a generalization of Theorem 1.3.

Theorem 3.8. Let $N, n \geqslant 1$ and $r_{1}, \ldots, r_{N} \in(0, \infty)$. Assume that $\phi: \mathcal{K}^{n} \rightarrow[0, \infty)$ satisfies $(a)$ and $(b)$. Let $h_{1}, \ldots, h_{N}$ be probability densities on $\mathbb{R}^{n}$ with $h_{i}(x)=$ $\prod_{j=1}^{n} h_{i j}\left(x_{j}\right)$ and each $h_{i j}$ is a probability density on $\mathbb{R}$ that is bounded by one. Consider independent random vectors $X_{1}, \ldots, X_{N}$ and $Y_{1}, \ldots, Y_{N}$ such that $X_{i}$ is distributed according to $h_{i}$ and $Y_{i}$ according to $\mathbb{1}_{Q_{n}}$, for $i=1, \ldots, N$. Then for any $s \geqslant 0$,

$$
\mathbb{P}\left(\phi\left(\bigcap_{i=1}^{N} B\left(X_{i}, r_{i}\right)\right)>s\right) \leqslant \mathbb{P}\left(\phi\left(\bigcap_{i=1}^{N} B\left(Y_{i}, r_{i}\right)\right)>s\right) .
$$

Proof. Note that each $h_{i j}^{*}$ is less peaked than $\mathbb{1}_{[-1 / 2,1 / 2]}$, hence by Theorem 3.4, $\prod_{i=1}^{N} \prod_{j=1}^{n} h_{i j}^{*}$ is less peaked than $\prod_{i=1}^{N} \mathbb{1}_{Q_{n}}$. Let $F$ be as in Lemma 3.6, $s>0$ and $H=\mathbb{1}_{\{F>s\}}$. For $x_{i} \in \mathbb{R}^{n}$ we write $x_{i}=\left(x_{i 1}, \ldots, x_{i n}\right)$. Since $F$ is even and quasi-concave on its support, we can apply Theorem 3.2 (considering $F$ as a quasi-concave function on $\mathbb{R}^{n N}$ as in Remark 3.3(i)) and Theorem 3.4 to obtain

$$
\begin{aligned}
\mathbb{P}( & \left.\phi\left(\bigcap_{i=1}^{N} B\left(X_{i}, r_{i}\right)\right)>s\right) \\
& =\int_{\mathbb{R}^{n}} \ldots \int_{\mathbb{R}^{n}} H\left(x_{1}, \ldots, x_{N}\right) \prod_{i=1}^{N} \prod_{j=1}^{n} h_{i j}\left(x_{i j}\right) d x_{1} \ldots d x_{N} \\
& \leqslant \int_{\mathbb{R}^{n}} \ldots \int_{\mathbb{R}^{n}} H\left(x_{1}, \ldots, x_{N}\right) \prod_{i=1}^{N} \prod_{j=1}^{n} h_{i j}^{*}\left(x_{i}\right) d x_{1} \ldots d x_{N}
\end{aligned}
$$




$$
\begin{aligned}
& \leqslant \int_{\mathbb{R}^{n}} \ldots \int_{\mathbb{R}^{n}} H\left(x_{1}, \ldots, x_{N}\right) \prod_{i=1}^{N} \mathbb{1}_{Q_{n}}\left(x_{i}\right) d x_{1} \ldots d x_{N} \\
& =\mathbb{P}\left(\phi\left(\bigcap_{i=1}^{N} B\left(Y_{i}, r_{i}\right)\right)>s\right) .
\end{aligned}
$$

Remark 3.9. One can adapt the latter argument to treat densities $h_{i j}$ that are not necessarily bounded by the same value. In this case, $h_{i j}^{*}$ is less peaked than $\left\|h_{i j}\right\|_{\infty} \mathbb{1}_{\left[-\frac{1}{2\left\|h_{i j}\right\|_{\infty}}, \frac{1}{\left.2\left\|h_{i j}\right\|_{\infty}\right]}\right.}$. Then the corresponding extremizers would be uniform measures on suitable coordinate boxes.

\section{Wulff shapes and ball-polyhedra}

Viewing a convex body as the intersection of its supporting halfspaces leads naturally to approximation by ball-polyhedra of large radii. More generally, one can work with Wulff shapes, which are defined as intersections of halfspaces. In this section, we make this connection explicit; detailed proofs are included for completeness and this will aid in interpreting the stochastic dominance discussed in the introduction. For background on Wulff shapes in BrunnMinkowski theory and further references, see Schneider [36, §7.5] and for recent results see work of Böröczky, Lutwak, Yang and Zhang [8] and Schuster and Weberndorfer [37].

If $f: S^{n-1} \rightarrow \mathbb{R}$ is a positive continuous function, the Wulff shape $W(f)$ is defined by

$$
W(f)=\bigcap_{\theta \in S^{n-1}} H^{-}(\theta, f(\theta)),
$$

where

$$
H^{-}(\theta, f(\theta))=\left\{x \in \mathbb{R}^{n}:\langle x, \theta\rangle \leqslant f(\theta)\right\} .
$$

Then $W(f)$ is a convex body with the origin in its interior. If $K$ is a convex body with positive support function $h_{K}$, then $W\left(h_{K}\right)=K$.

With $f$ as above and $R>\sup _{\theta \in S^{n-1}} f(\theta)$, we introduce a star body $A(f, R)$ by specifying its radial function:

$$
\rho_{A(f, R)}(-\theta)=R-f(\theta) \quad\left(\theta \in S^{n-1}\right) .
$$

The role of $A(f, R)$ is described in the next result; as above, $\operatorname{vr}(A(f, R))$ is the radius of a Euclidean ball with the same volume as $A(f, R)$. When $f$ is the (positive) support function $h_{K}$ of a convex body $K$ we also write $A(K, R)$ instead of $A\left(h_{K}, R\right)$. 
Proposition 4.1. Let $f: S^{n-1} \rightarrow \mathbb{R}$ be positive and continuous, $R>\sup _{\theta \in S^{n-1}} f(\theta)$ and $A(f, R)$ as in (4.3). Then, in the Hausdorff metric,

$$
W(f)=\lim _{R \rightarrow \infty} \bigcap_{x \in A(f, R)} B(x, R),
$$

and

$$
R-\operatorname{vr}(A(f, R)) \leqslant \int_{S^{n-1}} f(\theta) d \sigma(\theta) ;
$$

moreover, equality holds as $R \rightarrow \infty$.

The proof of the proposition relies on the following lemmas.

Lemma 4.2. Let $N, n \geqslant 1, x_{1}, \ldots, x_{N} \in \mathbb{R}^{n}$ and set $P=\operatorname{conv}\left\{x_{1}, \ldots, x_{N}\right\}$. Then for each $r>0$,

$$
\bigcap_{x \in P} B(x, r)=\bigcap_{i=1}^{N} B\left(x_{i}, r\right) .
$$

Proof of Lemma 4.2. Let $y \in \bigcap_{i=1}^{N} B\left(x_{i}, r\right)$ so that $\left|y-x_{i}\right| \leqslant r$ for each $i=1, \ldots, N$. Let $x \in P$ and write $x=\sum_{i=1}^{N} \alpha_{i} x_{i}$, where $\alpha_{1}, \ldots, \alpha_{N} \geqslant 0$ and $\sum_{i=1}^{N} \alpha_{i}=1$. Then

$$
|y-x|=\left|\sum_{i=1}^{N} \alpha_{i} y-\sum_{i=1}^{N} \alpha_{i} x_{i}\right| \leqslant \sum_{i=1}^{N} \alpha_{i}\left|y-x_{i}\right| \leqslant r
$$

hence $y \in \bigcap_{x \in P} B(x, r)$. The reverse inclusion is trivial.

Lemma 4.3. Let $f: S^{n-1} \rightarrow \mathbb{R}$ be positive and continuous. Assume that $\theta_{1}, \ldots, \theta_{N}$ are points on the sphere that do not lie on a hemisphere. Then

$$
\bigcap_{i=1}^{N} H^{-}\left(\theta_{i}, f\left(\theta_{i}\right)\right)=\lim _{R \rightarrow \infty} \bigcap_{i=1}^{N} B\left(-\left(R-f\left(\theta_{i}\right)\right) \theta_{i}, R\right),
$$

where the convergence is in the Hausdorff metric.

Proof of Lemma 4.3. Fix $R>\sup _{\theta \in S^{n-1}} f(\theta)$. Set $L=\bigcap_{i=1}^{N} H^{-}\left(\theta_{i}, f\left(\theta_{i}\right)\right)$. By definition of $A(f, R)$,

$$
\bigcap_{i=1}^{N} B\left(-\left(R-f\left(\theta_{i}\right)\right) \theta_{i}, R\right) \subseteq \bigcap_{i=1}^{N} H^{-}\left(\theta_{i}, f\left(\theta_{i}\right)\right)
$$

Next note that for any $\theta \in S^{n-1}$,

$$
\begin{aligned}
B(-(R-f(\theta)) \theta, R) & \supseteq\left\{x \in L:|x+(R-f(\theta)) \theta|^{2} \leqslant R^{2}\right\} \\
& =\left\{x \in L:\langle x, \theta\rangle \leqslant \frac{2 R f(\theta)-f^{2}(\theta)-|x|^{2}}{2(R-f(\theta))}\right\}
\end{aligned}
$$




$$
\begin{aligned}
& =\left\{x \in L:\langle x, \theta\rangle \leqslant \frac{1-\frac{f(\theta)}{2 R}-\frac{|x|^{2}}{2 R f(\theta)}}{1-\frac{f(\theta)}{R}} f(\theta)\right\} \\
& \supseteq\{x \in L:\langle x, \theta\rangle \leqslant(1-O(1 / R)) f(\theta)\},
\end{aligned}
$$

where the implied constants in $O(1 / R)$ depend only the extreme values of $f$ on $S^{n-1}$. Combining this with (4.8), we get

$$
(1-O(1 / R)) L \subseteq \bigcap_{i=1}^{N} B\left(-\left(R-f\left(\theta_{i}\right)\right) \theta_{i}, R\right) \subseteq L,
$$

which gives the result.

Proof of Proposition 4.1. The map $\theta \mapsto-(R-f(\theta)) \theta$ is a bijection between $S^{n-1}$ and the boundary $\partial A(f, R)$ of $A(f, R)$. Therefore

$$
\begin{aligned}
\bigcap_{\theta \in S^{n-1}} B(-(R-f(\theta)) \theta, R) & =\bigcap_{x \in \partial A(f, R)} B(x, R) \\
& =\bigcap_{x \in A(f, R)} B(x, R),
\end{aligned}
$$

where the last equality is simply Lemma 4.2 applied on each line segment

$$
P(\theta)=\operatorname{conv}\left\{\rho_{A(f, R)}(\theta) \theta,-\rho_{A(f, R)}(-\theta) \theta\right\} \quad\left(\theta \in S^{n-1}\right) .
$$

Thus equality (4.4) follows from Lemma 4.3. Since $A(f, R)$ is a star body, we can use polar coordinates and Jensen's inequality to get

$$
\begin{aligned}
\operatorname{vr}(A(f, R)) & =\left(\int_{S^{n-1}} \rho_{A(f, R)}(-\theta)^{n} d \sigma(\theta)\right)^{1 / n} \\
& =\left(\int_{S^{n-1}}(R-f(\theta))^{n} d \sigma(\theta)\right)^{1 / n} \\
& \geqslant R-\int_{S^{n-1}} f(\theta) d \sigma(\theta) .
\end{aligned}
$$

Writing $\|f\|_{1}=\int_{S^{n-1}} f(\theta) d \sigma(\theta)$, we can prove that asymptotic equality holds in the latter by Taylor expansion:

$$
\begin{aligned}
\operatorname{vr}(A(f, R)) & =R\left(\int_{S^{n-1}}\left(1-\frac{n f(\theta)}{R}+O\left(1 / R^{2}\right)\right) d \sigma(\theta)\right)^{1 / n} \\
& =R\left(1-\frac{n\|f\|_{1}}{R}+O\left(1 / R^{2}\right)\right)^{1 / n}
\end{aligned}
$$




$$
\begin{aligned}
& =R \exp \left(\frac{1}{n} \log \left(1-\frac{n\|f\|_{1}}{R}+O\left(1 / R^{2}\right)\right)\right) \\
& =R \exp \left(\frac{1}{n}\left(-\frac{n\|f\|_{1}}{R}+O\left(1 / R^{2}\right)\right)\right) \\
& =R \exp \left(-\frac{\|f\|_{1}}{R}+O\left(1 / R^{2}\right)\right) \\
& =R\left(1-\frac{\|f\|_{1}}{R}+O\left(1 / R^{2}\right)\right) \\
& =R-\|f\|_{1}+O(1 / R) .
\end{aligned}
$$

\section{Randomized inequalities related to the generalized Urysohn inequality}

In this section, we discuss and compare the randomized versions of the generalized Urysohn inequality. We start by sketching the proof of Böröczky and Schneider mentioned in the introduction. With $\mu_{K}$ and $\mathcal{H}_{K}$ as above, we have

$$
\mu_{K}(A)=\int_{S^{n-1}} \int_{0}^{1} \mathbb{1}_{\left\{H\left(\theta, h_{K}(\theta)+t\right) \in A\right\}} d t d \sigma(\theta)
$$

for Borel sets $A \subseteq \mathcal{H}_{K}$. For $\Theta=\left(\theta_{1}, \ldots, \theta_{N}\right) \in\left(S^{n-1}\right)^{N}$ and $t=\left(t_{1}, \ldots, t_{N}\right) \in \mathbb{R}^{N}$, set

$$
P(K, \Theta, t)=H^{-}\left(\theta_{1}, h_{K}\left(\theta_{1}\right)+t_{1}\right) \cap \ldots \cap H^{-}\left(\theta_{N}, h_{K}\left(\theta_{N}\right)+t_{N}\right) \cap(K+B) .
$$

Write $d \Theta$ for $d \sigma\left(\theta_{1}\right) \ldots d \sigma\left(\theta_{N}\right)$. For indepedent random hyperplanes $H_{1}, \ldots, H_{N}$ sampled according to $\mu_{K}$, set

$$
K^{(N)}=\bigcap_{i=1}^{N} H_{i}^{-} \cap(K+B) .
$$

Then

$$
\mathbb{E} V_{j}\left(K^{(N)}\right)^{1 / j}=\int_{\left(S^{n-1}\right)^{N}} \int_{[0,1]^{N}} V_{j}(P(K, \Theta, t))^{1 / j} d t d \Theta .
$$

For convex bodies $K$ and $L$ in $\mathbb{R}^{n}$ and $\alpha \in[0,1]$, one has the following inclusion

$$
(1-\alpha) P(K, \Theta, t)+\alpha P(L, \Theta, t) \subseteq P((1-\alpha) K+\alpha L, \Theta, t) .
$$


Thus

$$
V_{j}(P((1-\alpha) K+\alpha L, \Theta, t))^{1 / j} \geqslant(1-\alpha) V_{j}(P(K, \Theta, t))^{1 / j}+\alpha V_{j}(P(L, \Theta, t))^{1 / j} .
$$

Then

$$
\mathbb{E} V_{j}\left([(1-\alpha) K+\alpha L]^{(N)}\right)^{1 / j} \geqslant(1-\alpha) \mathbb{E} V_{j}\left(K^{(N)}\right)^{1 / j}+\alpha \mathbb{E} V_{j}\left(L^{(N)}\right)^{1 / j} .
$$

Thus, $K \mapsto \mathbb{E} V_{j}\left(K^{(N)}\right)^{1 / j}$ is concave with respect to Minkowski addition and it is also rotation invariant and continuous with respect to $\delta^{H}$. In particular, for any direction $u$, the Minkowski symmetral $M_{u}(K)=\frac{K+R_{u}(K)}{2}$ satisfies $\mathbb{E} V_{j}\left(M_{u}(K)^{(N)}\right)^{1 / j} \geqslant \mathbb{E} V_{j}\left(K^{(N)}\right)^{1 / j}$ (where, as above, $R_{u}$ denotes reflection about $\left.u^{\perp}\right)$. A theorem of Hadwiger (e.g., [36]) implies there is a sequence of directions so that successive Minkowski symmerizations about those directions converge to a Euclidean ball with the same mean width as $K$. This establishes (1.5).

Next, we prove the following extension of Corollary 1.2.

Corollary 5.1. Let $K$ be a convex body in $\mathbb{R}^{n}$ with the origin in its interior, $R>0$ and assume $K \subseteq B(0, R)$. Consider independent random vectors $X_{1}, \ldots, X_{N}$ sampled according to $\frac{1}{|A(K, R)|} \mathbb{1}_{A(K, R)}$ and $Z_{1}, \ldots, Z_{N}$ according to $\frac{1}{|r B|} \mathbb{1}_{r B}$, where $r=r(K, n, R)$ satisfies $|A(K, R)|=|r B|$. Let $\phi: \mathcal{K}^{n} \rightarrow(0, \infty)$ satisfy $(a),(b)$ and $(c)$ (as defined at the beginning of $\S 3)$. Then for each $p \in \mathbb{R}$,

$$
\left(\mathbb{E} \phi\left(\bigcap_{i=1}^{N} B\left(X_{i}, R\right)\right)^{p}\right)^{1 / p} \leqslant\left(\mathbb{E} \phi\left(\bigcap_{i=1}^{N} B\left(Z_{i}, R\right)\right)^{p}\right)^{1 / p} .
$$

Consequently, if $\phi$ is continuous with respect to $\delta^{H}$, we get

$$
\min _{x_{1}, \ldots, x_{N} \in A(K, R)} \phi\left(\bigcap_{i=1}^{N} B\left(x_{i}, R\right)\right) \leqslant \min _{z_{1}, \ldots, z_{N} \in r B} \phi\left(\bigcap_{i=1}^{N} B\left(z_{i}, R\right)\right) .
$$

Proof. Choose $\varepsilon>0$ such that $B(0, \varepsilon) \subseteq K$ so that for any $x_{1}, \ldots, x_{N} \in A(K, R)$, we have

$$
B(0, \varepsilon) \subseteq \bigcap_{i=1}^{N} B\left(x_{i}, R\right) \subseteq B\left(x_{1}, R\right) .
$$

In particular, all the moments in (5.1) are positive and finite. The same argument applies to points in $r B$. The moment inequality (5.1) follows immediately from Theorem 3.1. If $\phi$ is continuous, then

$$
\min _{x_{1}, \ldots, x_{N} \in A(K, R)} \phi\left(\bigcap_{i=1}^{N} B\left(x_{i}, R\right)\right)=\lim _{p \rightarrow-\infty}\left(\mathbb{E} \phi\left(\bigcap_{i=1}^{N} B\left(X_{i}, R\right)\right)^{p}\right)^{1 / p}
$$

and the same holds for $r B$ in place of $A(K, R)$ and $Z_{i}$ for $X_{i}$, which gives (5.2). 
For $N>n$ and $j \in\{1, \ldots, n\}$, let

$$
m_{j, N}(K)=\min \left\{V_{j}\left(\bigcap_{i=1}^{N} H_{i}^{-}\right): K \subseteq H_{i}^{-}, i=1, \ldots, N\right\}
$$

where $H_{i}^{-}$is the closed halfspace bounded by $H_{i}$ that contains $K$. As a consequence of Corollary 5.1, we get the following, which is a special case of a result due to Schneider [35].

Corollary 5.2. Let $K$ be a convex body in $\mathbb{R}^{n}, N>n$ and $j \in\{1, \ldots, n\}$. Then

$$
m_{j, N}(K) \leqslant m_{j, N}((w(K) / 2) B) .
$$

Proof. Without loss of generality we will assume that the origin is an interior point of $K$. Choose $R>0$ such that $K \subseteq B(0, R)$. Since $A(K, R)$ is star-shaped, for any $x \in A(K, R)$ the line through $x$ and the origin intersects $A(K, R)$ in a line segment, the endpoints of which are on the boundary $\partial A(K, R)$. Hence quasiconcavity of $V_{j}$ yields

$$
\min _{x_{1}, \ldots, x_{N} \in A(K, R)} V_{j}\left(\bigcap_{i=1}^{N} B\left(x_{i}, R\right)\right)=\min _{x_{1}, \ldots, x_{N} \in \partial A(K, R)} V_{j}\left(\bigcap_{i=1}^{N} B\left(x_{i}, R\right)\right) ;
$$

the same holds with $r B$ in place of $A(K, R)$. Therefore, (5.2) implies

$$
\min _{x_{1}, \ldots, x_{N} \in \partial A(K, R)} V_{j}\left(\bigcap_{i=1}^{N} B\left(x_{i}, R\right)\right) \leqslant \min _{x_{1}, \ldots, x_{N} \in r S^{n-1}} V_{j}\left(\bigcap_{i=1}^{N} B\left(x_{i}, R\right)\right),
$$

hence

$$
\min _{\theta_{1}, \ldots, \theta_{N} \in S^{n-1}} V_{j}\left(\bigcap_{i=1}^{N} B\left(-\left(R-h_{K}\left(\theta_{i}\right)\right) \theta_{i}, R\right)\right) \leqslant \min _{x_{1}, \ldots, x_{N} \in r S^{n-1}} V_{j}\left(\bigcap_{i=1}^{N} B\left(x_{i}, R\right)\right) .
$$

By (4.9), we have

$$
m_{j, N}(K)=\sup _{R>0} \min _{\theta_{1}, \ldots, \theta_{N} \in S^{n-1}} V_{j}\left(\bigcap_{i=1}^{N} B\left(-\left(R-h_{K}\left(\theta_{i}\right) \theta_{i}, R\right)\right) .\right.
$$

For $K=(w(K) / 2) B$, we get

$$
\begin{aligned}
m_{j, N}((w(K) / 2) B) & =\sup _{R>0} \min _{\theta_{1}, \ldots, \theta_{N} \in S^{n-1}} V_{j}\left(\bigcap_{i=1}^{N} B\left(-(R-w(K) / 2) \theta_{i}, R\right)\right) \\
& =\sup _{R>0} \min _{\theta_{1}, \ldots, \theta_{N} \in S^{n-1}} V_{j}\left(\bigcap_{i=1}^{N} B\left(-r(K, n, R) \theta_{i}, R\right)\right),
\end{aligned}
$$

where the latter follows from the asymptotic equality in (4.5). The corollary now follows from (5.8) and (5.9). 
When $N \rightarrow \infty$ in (5.5), we get (1.2). This indicates that the stochastic dominance in Theorem 1.1 for $f=\frac{1}{|A(K, R)|} \mathbb{1}_{A(K, R)}$ can be regarded as a distributional form of (1.2).

Remark 5.3. Schneider [35] proved a more general variant of (5.5) with $V_{j}$ replaced by a function $\phi$ which is concave, monotone, upper semi-continuous and minimized over rotations of $K$. We do not pursue a more detailed comparison as this is not our main goal.

Schneider's result (5.5) is a companion to that of Macbeath for maximum volume simplices inscribed in convex bodies [27]. Using (5.5) for $j=n$ and the reverse Urysohn inequality due to Pisier [32] and Figiel and TomczakJaegermann [16] we get the following.

Corollary 5.4. Let $K$ be a convex body in $\mathbb{R}^{n}$. Then there is a simplex $S$ containing K such that

$$
V_{n}(S) \leq(C \log n)^{n} n^{\frac{n+1}{2}} V_{n}(K),
$$

where $C$ is an absolute constant.

The latter improves on a result of Kanazawa [23] who proved that $V_{n}(S) \leq$ $n^{n-1} V_{n}(K)$, which extends a classical planar result of Gross [20] to higher dimensions.

Proof. As the problem is invariant under affine transformations, we may first apply the reverse Urysohn inequality due to Pisier and Figiel and TomczakJaegermann (see [2, Theorem 6.5.4]) and assume that

$$
\frac{w(K)}{w(B)} \leqslant C_{1} \log n\left(\frac{V_{n}(K)}{V_{n}(B)}\right)^{1 / n},
$$

where $C_{1}$ is an absolute constant. By Schneider's result (5.5) we have

$$
m_{n, n+1}(K) \leqslant m_{n, n+1}(B)\left(\frac{w(K)}{w(B)}\right)^{n} .
$$

On the other hand,

$$
m_{n, n+1}(B)=\frac{n^{\frac{n}{2}}(n+1)^{\frac{n+1}{2}}}{n !} .
$$

The result now follows from (5.12) and (5.13). 


\subsection{Further connections to Minkowski symmetrization}

In this section, we discuss the effect of Minkowski symmetrization of $K$ on $A(K, R)$. We show that one can obtain (5.2) via Minkowski symmetrization as well. If $K$ and $L$ are convex bodies, the equality $h_{(K+L) / 2}=\left(h_{K}+h_{L}\right) / 2$ implies

$$
\rho_{A\left(\frac{K+L}{2}, R\right)}=\frac{1}{2}\left(\rho_{A(K, R)}+\rho_{A(L, R)}\right) .
$$

In other words, the map $K \mapsto A(K, R)$ takes Minkowski sums to radials sums. In particular, if $u \in S^{n-1}$ and $M_{u}(K)$ is the Minkowski symmetral of $K$ about $u^{\perp}$, then $A\left(M_{u}(K), R\right)$ is the star-body with radial function $\frac{1}{2}\left(\rho_{A(K, R)}+\rho_{A\left(R_{u}(K), R\right)}\right)$.

Assume now that $\theta_{1}, \ldots, \theta_{N} \in S^{n-1}$. Then

$$
\begin{aligned}
\bigcap_{i=1}^{N} B\left(-\left(R-h_{M_{u}(K)}\left(\theta_{i}\right) \theta_{i}, R\right)\right. \\
\quad=\bigcap_{i=1}^{N} B\left(\rho_{A\left(M_{u}(K), R\right)}\left(\theta_{i}\right) \theta_{i}, R\right) \\
\supseteq \supseteq \frac{1}{2} \bigcap_{i=1}^{N} B\left(\rho_{A(K, R)}\left(\theta_{i}\right) \theta_{i}, R\right)+\frac{1}{2} \bigcap_{i=1}^{N} B\left(\rho_{A\left(R_{u}(K), R\right)}\left(\theta_{i}\right) \theta_{i}, R\right) \\
\quad=\frac{1}{2} \bigcap_{i=1}^{N} B\left(-\left(R-h_{K}\left(\theta_{i}\right) \theta_{i}\right), R\right)+\frac{1}{2} \bigcap_{i=1}^{N} B\left(-\left(R-h_{R_{u}(K)}\left(\theta_{i}\right)\right) \theta_{i}, R\right) \\
\quad=\frac{1}{2} \bigcap_{i=1}^{N} B\left(-\left(R-h_{K}\left(\theta_{i}\right) \theta_{i}\right), R\right)+\frac{1}{2} R_{u}\left(\bigcap_{i=1}^{N} B\left(-\left(R-h_{K}\left(R_{u}^{t} \theta_{i}\right)\right) R_{u}^{t} \theta_{i}, R\right)\right),
\end{aligned}
$$

where $R_{u}^{t}$ is the transpose of $R_{u}$. We now use quasi-concavity of $\phi$ and rotation invariance to get

$$
\phi\left(\bigcap_{i=1}^{N} B\left(-\left(R-h_{M_{u}(K)}\left(\theta_{i}\right)\right) \theta_{i}, R\right)\right) \geqslant \min _{\theta_{1}, \ldots, \theta_{N} \in S^{n-1}} \phi\left(\bigcap_{i=1}^{N} B\left(-\left(R-h_{K}\left(\theta_{i}\right)\right) \theta_{i}, R\right)\right) .
$$

As mentioned above, given a convex body $K$, a theorem of Hadwiger implies that there is a sequence of directions so that successive Minkowski symmetrizations about those directions converge to a Euclidean ball with the same mean width as $K$. Combining this with inequality (5.14), we get another proof of (5.2).

\subsection{Connection between random ball-polyhedra and random convex hulls}

As mentioned already, the inequality for random ball-polyhedra obtained by taking $j=n$ in (1.6) implies Urysohn's inequality, and so does the inequality for random convex hulls when $j=1$ in (1.4). Here we show that the former 
implies the latter. The proof uses the following theorem of Gorbovickis [18, Theorem 4].

Theorem 5.5. Let $x_{1} \ldots, x_{N} \in \mathbb{R}^{n}$ where $n \geqslant 2$. Then the following asymptotic equality holds as $R \rightarrow \infty$ :

$$
\left|\left(\bigcap_{i=1}^{N} B\left(x_{i}, R\right)\right)\right|=\omega_{n} R^{n}-n \omega_{n} w\left(\operatorname{conv}\left\{x_{1}, \ldots, x_{N}\right\}\right) R^{n-1}+o\left(R^{n-1}\right) .
$$

Assume that $K$ is a convex body in $\mathbb{R}^{n}$ with $|K|=|B|$. Sample independent random vectors $X_{1}, \ldots, X_{N}$ in $K$ and $Z_{1}, \ldots, Z_{N}$ in $B$ according to their respective uniform probability measures. For each fixed value of $X_{1}, \ldots, X_{N}$, Theorem 5.5 implies

$$
n \omega_{n} w\left(\operatorname{conv}\left\{X_{1}, \ldots, X_{N}\right\}\right)=R-R^{-(n-1)}\left|\left(\bigcap_{i=1}^{N} B\left(X_{i}, R\right)\right)\right|+o(1),
$$

as $R \rightarrow \infty$. By compactness of $K$, we can use dominated convergence to conclude

$$
n \omega_{n} \mathbb{E} w\left(\operatorname{conv}\left\{X_{1}, \ldots, X_{N}\right\}\right)=R-R^{-(n-1)} \mathbb{E}\left|\left(\bigcap_{i=1}^{N} B\left(X_{i}, R\right)\right)\right|+\mathbb{E} o(1),
$$

as $R \rightarrow \infty$. By continuity of the volume of the intersection and the mean width, the quantity $\mathbb{E} o(1)$ is also of the form $o(1)$. The same argument applies to $Z_{1}, \ldots, Z_{N}$. By Theorem 3.1, we get

$$
\mathbb{E} w\left(\operatorname{conv}\left\{X_{1}, \ldots, X_{N}\right\}\right) \geqslant \mathbb{E} w\left(\operatorname{conv}\left\{Z_{1}, \ldots, Z_{N}\right\}\right),
$$

which is equivalent to the $j=1$ case in (1.4).

\section{Acknowledgements}

It is our pleasure to thank Rolf Schneider for helpful correspondence. We also thank Beatrice-Helen Vritsiou for helpful comments on a previous draft of this paper. Lastly, we thank the anonymous referee for careful reading and comments which improved the results and presentation of the paper.

\section{References}

[1] G. Ambrus, P. Kevei, and V. Vígh, The diminishing segment process, Statist. Probab. Lett. 82 (2012), no. 1, 191-195. 
[2] S. Artstein-Avidan, A. Giannopoulos, and V. D. Milman, Asymptotic geometric analysis. Part I, Mathematical Surveys and Monographs, vol. 202, American Mathematical Society, Providence, RI, 2015.

[3] K. Ball, Convex geometry and functional analysis, Handbook of the geometry of Banach spaces, Vol. I, North-Holland, Amsterdam, 2001, pp. 161-194.

[4] F. Barthe, Mesures unimodales et sections des boules $B_{p}^{n}$, C. R. Acad. Sci. Paris Sér. I Math. 321 (1995), no. 7, 865-868.

[5] _ Extremal properties of central half-spaces for product measures, J. Funct. Anal. 182 (2001), no. 1, 81-107.

[6] K. Bezdek, Lectures on sphere arrangements-the discrete geometric side, Fields Institute Monographs, vol. 32, Springer, New York; Fields Institute for Research in Mathematical Sciences, Toronto, ON, 2013.

[7] K. Bezdek, Z. Lángi, M. Naszódi, and P. Papez, Ball-polyhedra, Discrete Comput. Geom. 38 (2007), no. 2, 201-230.

[8] K. J. Böröczky, E. Lutwak, D. Yang, and G. Zhang, The log-BrunnMinkowski inequality, Adv. Math. 231 (2012), no. 3-4, 1974-1997.

[9] K. J. Böröczky and R. Schneider, The mean width of circumscribed random polytopes, Canad. Math. Bull. 53 (2010), no. 4, 614-628.

[10] H. J. Brascamp, E. H. Lieb, and J. M. Luttinger, A general rearrangement inequality for multiple integrals, J. Functional Analysis 17 (1974), 227-237.

[11] H. Busemann, Volume in terms of concurrent cross-sections, Pacific J. Math. 3 (1953), 1-12.

[12] S. Campi and P. Gronchi, Extremal convex sets for Sylvester-Busemann type functionals, Appl. Anal. 85 (2006), no. 1-3, 129-141.

[13] M. Christ, Estimates for the k-plane transform, Indiana Univ. Math. J. 33 (1984), no. 6, 891-910.

[14] D. Cordero-Erausquin, M. Fradelizi, G. Paouris, and P. Pivovarov, Volume of the polar of random sets and shadow systems, Math. Ann. 362 (2015), no. 34, 1305-1325.

[15] B. Csikós, On the volume of the union and intersection of random balls, Audio talk and slides available at https://www.fields.utoronto.ca/audio/11-12/wksp_sphere/csikos. 
[16] T. Figiel and N. Tomczak-Jaegermann, Projections onto Hilbertian subspaces of Banach spaces, Israel J. Math. 33 (1979), no. 2, 155-171.

[17] F. Fodor, P. Kevei, and V. Vígh, On random disc polygons in smooth convex discs, Adv. in Appl. Probab. 46 (2014), no. 4, 899-918.

[18] I. Gorbovickis, Strict Kneser-Poulsen conjecture for large radii, Geom. Dedicata 162 (2013), 95-107.

[19] H. Groemer, On the mean value of the volume of a random polytope in a convex set, Arch. Math. (Basel) 25 (1974), 86-90.

[20] W. Gross, Über affine geometrie xiii, eine minimumeigenshaft der ellipse und des ellipsoids, Leipziger Berichte 70 (1918), 38-54.

[21] H. Hadwiger, Vorlesungen über Inhalt, Oberfläche und Isoperimetrie, Springer-Verlag, Berlin-Göttingen-Heidelberg, 1957.

[22] M. Hartzoulaki and G. Paouris, Quermassintegrals of a random polytope in a convex body, Arch. Math. (Basel) 80 (2003), no. 4, 430-438.

[23] A. Kanazawa, On the minimal volume of simplices enclosing a convex body, Arch. Math. (Basel) 102 (2014), no. 5, 489-492.

[24] M. Kanter, Unimodality and dominance for symmetric random vectors, Trans. Amer. Math. Soc. 229 (1977), 65-85.

[25] E. H. Lieb and M. Loss, Analysis, second ed., Graduate Studies in Mathematics, vol. 14, American Mathematical Society, Providence, RI, 2001.

[26] E. Lutwak and G. Zhang, Blaschke-Santaló inequalities, J. Differential Geom. 47 (1997), no. 1, 1-16.

[27] A. M. Macbeath, An extremal property of the hypersphere, Proc. Cambridge Philos. Soc. 47 (1951), 245-247.

[28] G. Paouris and P. Pivovarov, A probabilistic take on isoperimetric-type inequalities, Adv. Math. 230 (2012), no. 3, 1402-1422.

[29] Small-ball probabilities for the volume of random convex sets, Discrete Comput. Geom. 49 (2013), no. 3, 601-646.

[30] R. E. Pfiefer, The extrema of geometric mean values, ProQuest LLC, Ann Arbor, MI, 1982, Thesis (Ph.D.)-University of California, Davis. 
[31] , The historical development of J. J. Sylvester's four point problem, Math. Mag. 62 (1989), no. 5, 309-317.

[32] G. Pisier, Holomorphic semigroups and the geometry of Banach spaces, Ann. of Math. (2) 115 (1982), no. 2, 375-392.

[33] C. A. Rogers, A single integral inequality, J. London Math. Soc. 32 (1957), 102-108.

[34] C. A. Rogers and G. C. Shephard, Some extremal problems for convex bodies, Mathematika 5 (1958), 93-102.

[35] R. Schneider, Eine allgemeine Extremaleigenschaft der Kugel, Monatsh. Math. 71 (1967), 231-237.

[36] __ Convex bodies: the Brunn-Minkowski theory, expanded ed., Encyclopedia of Mathematics and its Applications, vol. 151, Cambridge University Press, Cambridge, 2014.

[37] F. E. Schuster and M. Weberndorfer, Volume inequalities for asymmetric Wulff shapes, J. Differential Geom. 92 (2012), no. 2, 263-283. 